\title{
An Interpretation of Behavior of Ethylamine Ion Produced
}

\author{
by Electron-impact"
}

\author{
HIROSHI ICHIKAWA* AND MICHIHIKO OGATA*
}

(Received 11 November 1975)

\begin{abstract}
The relative bond energies of the ethylamine ion and the potential energy curves of its dissociation were calculated by the unrestricted MINDO/2 method in attempt to explain the specific cleavage of the ion. Simple cleavage reactions of doublet ion were generally classified into two groups of reaction according to the formal movement of the unpaired electron in the reaction. It was revieled that the reactions so-called $\alpha$-cleavage or $\beta$-bond cleavage belong to a group of reaction in which a strong interstate interaction inevitablly appears in the course of reaction causing lower transition energy. A qualitative interpretation was given for the relationship between the net charge density of the highest occupied molecular orbital and the scission probabilities. The validity of the Bell-Evans-Polanyi principle in mass spectral reaction was discussed.
\end{abstract}

Organic chemical approaches to the interpretation of organic mass spectra have provided some simple empirical rules of breakdown processes $^{2 \sim 4)}$, which are here reffered to as the fragmentation rules. These rules have been found to be not only appricable in explaining the major fragmentation paths of organic molecular ion but also of special interest for organic chemists in the relationship between mass spectrometric, thermolytic, and photolytic fragmentation reactions ${ }^{5)}$, since the fragmentation rules can be regarded as a chemical reaction of a special electronic state of molecule, i. e., the state of cation radical. Thus, it is of our interest that the fundamental studies on the fragmentation rules may contribute not only to the establishment of the theory of mass spectral fragmentation but also to that of the correlation between the electronic state and its chemical behavior.

The prediction of intensities of spectra, whether produced by photons or by electrons, is still largely an unsolved theoretical problem. Theoretical studies of mass spectra have been made to give two divergent lines of research, the quasi-equilibrium theory $(\mathrm{QET})^{6 \sim 8)}$ and molecular-orbital-theoretical treatment (MOT) ${ }^{9 \sim 13)}$. Neither of these approaches seems to be readily adaptable to the interpretation of the fragmentation rules. The QET has clearly accounted for the fragmentation of small hydrocarbons and may be widely admitted to be true as a concept of fragmentation. However, it involves tremendous difficulties when applied to large molecular ions.

A number of papers has appeared discusing the relationship between the scission probability and the net charge density of the highest occupied molecular orbital (HOMO) obtained from the calculation for molecule ${ }^{9 \sim(3)}$. The assumption has been valid to predict the experimental results in many cases. There rises,

* Hoshi College of Pharmacy, 2-4 Ebara, Shinagawa-ku, Tokyo 142, Japan 
however, a simple question; why such an HOMO of molecule regulates the cleavage reaction in spite of the fact that the bond strength is governed by not only the electrons of the HOMO but also by all included in the molecular ion. Lorquet severely criticized this assumption ${ }^{14}$.15). However, as widely shown by Hirota et al. ${ }^{10 \sim 13)}$ the good agreement of this assumption with experimental results does not seem to be those of incident.

Being roughly classified, organic chemical fragmentation rules, the quasi-equilibrium theory, and the MO theory are prevalent in the treatment of the reactions of molecular ion and the some fruitful results have been obtained in their own terms. However, the relationship between them seems not to be given. In this paper, we will discuss the fundamental problems of the fragmentation on the ethylamine ion in attempt to obtain a qualitative interpretation for the relationship between the fragmentation rules, the MOT, and the QET.

\section{Electronic State of Reactive Molecular Ion.}

First we must define the electronic state of the molecular ion at the reactive state, since it may characterize the mode of fragmentation.

Although the electronic state of molecular ion is not defined in the fragmentation rules of organic mass spectra, they successfully explain major fragmentation paths of molecular ion in terms of electronic structure ${ }^{2 \sim 4)}$; it may be an important implication that the rules have been established basing on observation of metastable peaks. In our earlier paper ${ }^{16)}$, we have investigated the electronic structures of simple amine and alcohol ions pointing out that the electronic structures assumed by the fragmentation rules well accord with those of the ground states of molecular ion.

Tsuchiya studied the mass spectra of fragments with excess kinetic energy (KE mass spectra) ${ }^{177}$. The KE mass spectra forms when the energy of impinging electron is more than. $30 \mathrm{eV}$. And therefore, the KE ions may have far larger internal energy than ordinary, ions; this suggests that the molecular ions which produce the $\mathrm{KE}$ ions have large possibility of electronic excitation at the reactive state. This is strongly supported by the fact that the patterns of $\mathrm{KE}$ mass spectra appear to be quite different from those of ordinary mass spectra. Since the amounts of the $\mathrm{KE}$ ions are shown to be less than $1 \%$ of the total amounts of ions formed by electron-impact, most (more than $99 \%$ ) of the ions are considered to consist of the ground state ions. And therefore, it is our standpoint that any fact concerning the fragmentation rules, the QET, and the MOT should be interpreted in terms of the ground state molecular ion.

\section{The Relative Bond Energies of the Intact}

Molecular Ion. The fragmentation of the ethylamine ion involves the most of the fundamental problems of mass spectral reaction and has often appeared in discussions ${ }^{2,3,4.13 .18)}$ The well-established fact is that the process,

$$
\left[\mathrm{H}_{2} \mathrm{~N}-\mathrm{CH}_{2}-\mathrm{CH}_{3}\right]^{+\cdot} \longrightarrow \mathrm{H}_{2} \mathrm{~N}^{+}=\mathrm{CH}_{2}+\cdot \mathrm{CH}_{3}
$$

is extremely important with respect to other cleavages such as,

$$
\left[\mathrm{H}_{2} \mathrm{~N}-\mathrm{CH}_{2}-\mathrm{CH}_{3}\right]^{+\cdot} \longrightarrow \mathrm{H}_{2} \mathrm{~N} \cdot+\mathrm{CH}_{2} \mathrm{CH}_{3}{ }^{+}
$$

A simple approach to this problem may be to calculate the "bond energies" and to find the correlation between the scission probabilities and them. The total energy (E) calculated by such semiempirical MO methods as CNDO (Complete Neglect of Differential Overlap) ${ }^{(9,20)}$, INDO (Intermediate Neglect of Differential Overlap) ${ }^{211}$, and MINDO (Modified INDO) methods ${ }^{22}{ }^{23)}$ which neglect the three- and fourcenter interactions can be written as the sum 
of the one- $\left(\mathrm{E}_{\mathrm{A}}\right)$ and the two-center $\left(\mathrm{E}_{\mathrm{AB}}\right)$ energy terms,

$$
\mathrm{E}=\Sigma \mathrm{E}_{\mathrm{A}}+\sum_{\mathrm{A}>\mathrm{B}} \mathrm{E}_{\mathrm{AB}} \text {. }
$$

As Fischer and Kollmar pointed out ${ }^{24)}$, the twocenter term $\left(E_{A B}\right)$ represents the relative bond energy between atoms $\mathrm{A}$ and $\mathrm{B}$. In our previous paper of this series, we calculated the relative bond energies of simple molecular ions in attempt to predict the position of bond scission in terms of bond energy. This kind of approach has been unsuccessful in the extended Hückel $^{25)}$, unrestricted CNDO/2 (CNDO version 2), and INDO methods ${ }^{16)}$ These MO methods have been, however, discussed to be inadequate in the estimation of molecular energies $^{26)}$. In order to remove this difficulties, we calculated the $E_{A B}$ values of the ethylamine ion by the unrestricted MINDO/2 method which we have developed and proved to be best fitted in the estimation of energies of radicals ${ }^{27}$. The results are shown in Table 1, where in the first column the $E_{A B}$ values of the ethylamine molecule and in the second column those of its ion. The results are much the same as those in the CNDO/2 and INDO methods; removal of one electron from the HOMO of the neutral molecule weakens the $\mathrm{C}-\mathrm{C}$ bond but too slightly to expalin the predominance of the C-C bond-scission. Moreover configurational change of $\mathrm{NH}_{2}$ group from $\mathrm{sp}^{3}$ to $\mathrm{sp}^{2}$ does not effect any significant variations of bond energies. Therefore, it may be concluded that there is not any correlation between the predominance of bond scission and the relative bond energy; this will be rationalized in the later section.

The Potential Energy Curves of the Dissociation and the Changes of the Unpaired Electron in the C-N and C-C Cleavage of the Ground-State Ethylamine Ion. The activation energy and heat of reaction may be obtained if the total energies are drawn as a function of the distance of two dissociating fragments. Investigation of the electronic structures in the process of dissociation may reveal the role of the unpaired electron to the dissociation. Lorquet et al. calculated the potential energy curves and the net charge densities in the dissociation of the ethylamine ion by the CNDO/2 method $^{(5)}$. The molecular energy is critically dependent on the used MO method even if one tries to obtain the relative values. As mentioned, the CNDO $/ 2$ method, unfortunately, is known not to be well adapted for the purpose even if the relative values are in question ${ }^{26)}$. Besides, they did not give any interpretation on the role of the unpaired electron. As we already pointed out in our earlier paper ${ }^{16)}$, the net charge is generally distributed over the whole system and is not very characteristic, and therefore, the change of

Table 1. Relative Bond Energies of the Ethylamine ion by the MINDO/2 Method (a. u.).

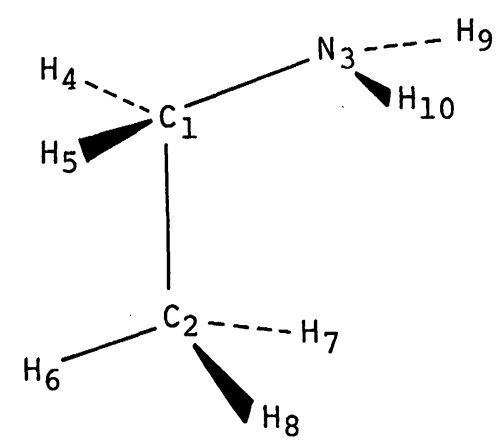

\begin{tabular}{ccc}
\hline Bond & Molecule & Ion \\
\hline $\mathrm{C}_{1}-\mathrm{C}_{2}$ & -0.5284 & -0.4938 \\
$\mathrm{C}_{1}-\mathrm{N}_{3}$ & -0.5486 & -0.4729 \\
$\mathrm{C}_{1}-\mathrm{H}_{4}$ & -0.4679 & -0.4637 \\
$\mathrm{C}_{2}-\mathrm{H}_{6}$ & -0.4624 & -0.4631 \\
$\mathrm{C}_{2}-\mathrm{H}_{7}$ & -0.4610 & -0.4634 \\
$\mathrm{~N}_{3}-\mathrm{H}_{9}$ & -0.5446 & -0.4984 \\
\hline
\end{tabular}




\section{H. Ichikawa and M. Ogata}

distribution of the unpaired electron should be refered to in order to interpret the role of the unpaired electron in the fragmentation.

Recalculations have been carried out by the unrestricted MINDO/2 method $^{27)}$ without including the effects of configuration interactions (Cl's) for the sake of simplicity in calculation.

Because of the difficulty of obtaining the rigorous potential energy, we adapted an approximate method as follows; the carbon and the nitrogen atoms in the intact molecular ion are considered to have the $\mathrm{sp}^{3}$ configurations similar to the molecule while after the complete dissociation those atoms must have the $\mathrm{sp}^{2}$ configurations. If an adiabatic process holds in the dissociation, it may be a nature of reaction that the $\mathrm{sp}^{3}$ configurations of the nitrogen and carbon atoms gradually change to $\mathrm{sp}^{2}$ as the reaction proceeds. Thus the curve beneath the vertical potential energy curves for $\mathrm{sp}^{3}$ and $\mathrm{sp}^{2}$ configurations is considered to be close to the real potential energy curve. Figure 1 shows the potential energy curves for the $\mathrm{C}-\mathrm{N}$ and $\mathrm{C}-\mathrm{C}$ dissociations obtained by this method, where the conformations of the $\mathrm{CH}_{2}$ and $\mathrm{CH}_{3}$ groups are set to be staggered and where trans and cis represent the cases that the direction of the orbital of the unpaired electron is trans to the $\mathrm{C}-\mathrm{C}$ bond and is perpendicular to the molecular plane, respectively.

The difference of the potential energies between the $\mathrm{C}-\mathrm{N}$ and $\mathrm{C}-\mathrm{C}$ (trans) bonds around the bond-length of $2.0 \dot{\mathrm{A}}$ is calculated out to be about $20 \mathrm{kcal} / \mathrm{mol}$ and may be more realistic than Lorquet's result $(4.6 \mathrm{kcal} / \mathrm{mol})^{15}$. The absolute values obtained here are rather consistent with experiment; the experimental activation energies for the C-N and C-C dissociations are found to be $4.2^{28)}$ and $0.9 \mathrm{eV}^{29)}$, respectively. Our results are; 4.2 and $2.7 \mathrm{eV}$ for the $\mathrm{C}-\mathrm{N}$ and $\mathrm{C}-\mathrm{C}$ dissociations, respectively. The disagreement of the latter dissociation will be explained in the later section in terms of the degree of interstate interactions.

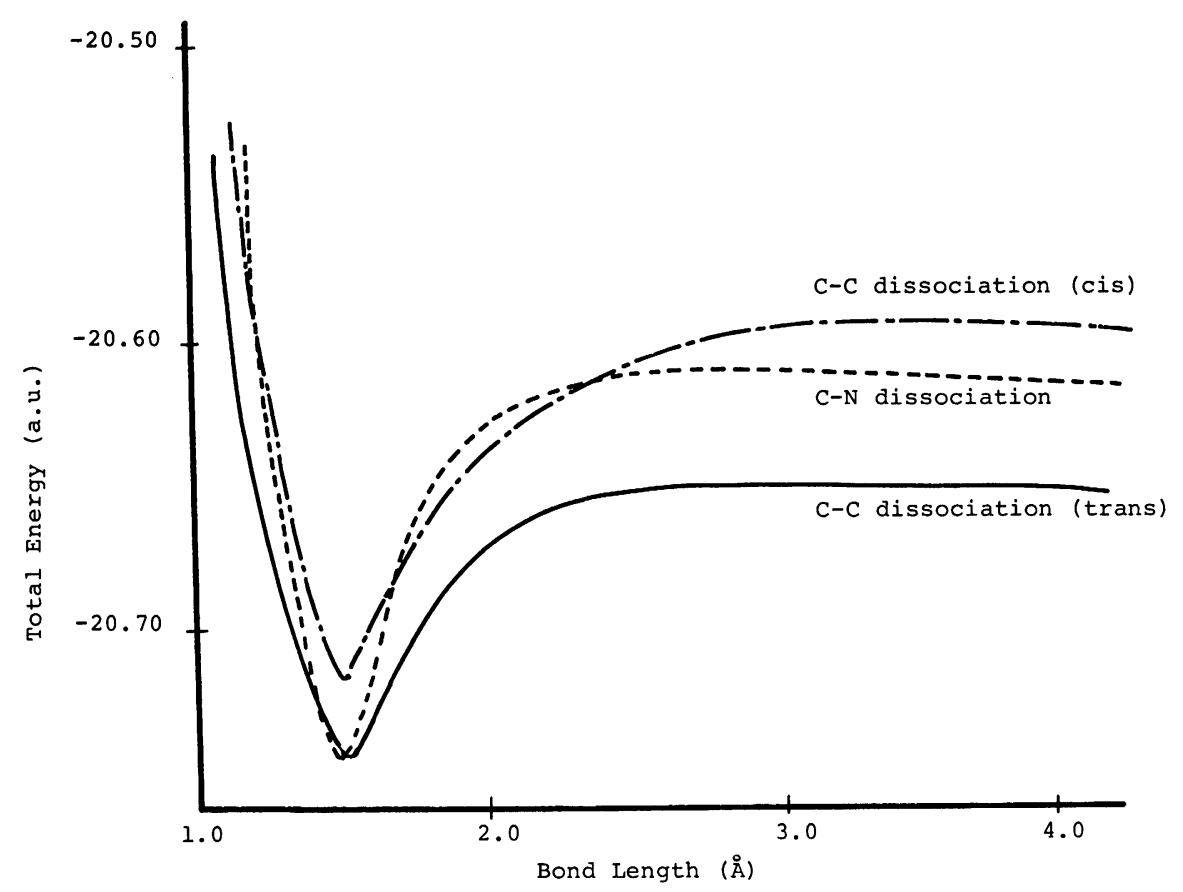

Fig. 1. Potential energy curves for the C-C and C-N dissociations of the ethylamine ion (MINDO/2). 
We can suppose without loss of generality that the unpaired electron has the $\alpha$-spin; the spin density $\left(\mathrm{D}^{\text {spin }} ; \mathrm{D}^{\text {spin }}=\mathrm{D}^{\alpha}-\mathrm{D}^{\beta}\right.$, where $\mathrm{D}^{\prime}$ and $\mathrm{D}^{\beta}$ are the electron densities contributed by the $\alpha$-spin and $\beta$-spin elentrons, respectively) may represents the distribution of the unpaired electron. The changes of the distribution of the unpaired electron and the relative bond-energies of the $\mathrm{C}-\mathrm{N}$ and $\mathrm{C}-\mathrm{C}$ bonds as the functions of the $\mathrm{C}-\mathrm{C}$ bond-length are shown in Fig. 2. When the $\mathrm{C}-\mathrm{N}$ bond is dissociated, the location of the unpaired electron remains unchanged giving neutral fragment, $\mathrm{H}_{2} \mathrm{~N}$, and cationic $\mathrm{CH}_{2} \mathrm{CH}_{3}$; the cleavage should be ionic and depicted organicchemically as,

$$
\mathrm{H}_{2} \stackrel{\hat{\mathrm{N}}}{-} \mathrm{CH}_{2}-\mathrm{CH}_{3} \longrightarrow \mathrm{H}_{2} \mathrm{~N} \cdot+{ }^{+} \mathrm{CH}_{2}-\mathrm{CH}_{3} \text {. }
$$

The relative bond energies $\left(\mathrm{E}_{\mathrm{AB}}\right)$ are about -1.04 and -1.70 for single $\mathrm{C}-\mathrm{N}$ and double $\mathrm{C}=\mathrm{N}$ bonds, respectively (the examples are ethylamine and formimide in their neutral molecules, respectively). When the $\mathrm{C}-\mathrm{C}$ bond of the trans conformation is dissociated, it will be seen that the unpaired electron which is at first localized on the aminoethylene group formally moves to the methyl site and that the $\mathrm{C}-\mathrm{N}$ bond becomes near double bond. This indicates that when the $\mathrm{C}-\mathrm{C}$ bond in the trans conformation is dissociated, the unpaired electron significantly participates in the formation of the $\mathrm{CN}$ double bond to cause the potential energy lower than that in the $\mathrm{C}-\mathrm{N}$ dissociation, since the potential energy curve of cis is as high as that in the $\mathrm{CN}$ dissociation (Fig. 1).

The interesting difference of the potential energy between the cis and trans conformations suggests that existence of stereospecificity in the fragmentation of the derivatives of ammonia.

That is, in the conformationally rigid system, the alkyl group attached to the $\alpha$-carbon atom must lose more easily when the bond is trans to the lone pair orbital than when it is cis.

\section{A Classification of the Cleavage Reactions of}

Doublet Ion. As seen in the dissociation of the

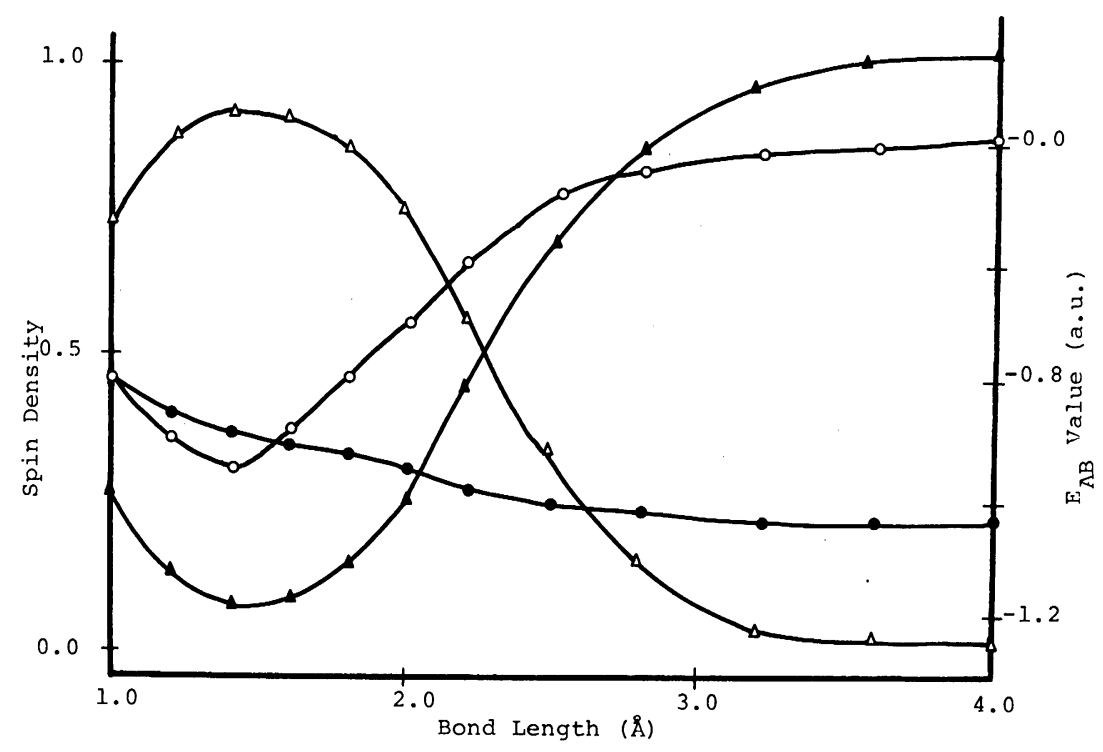

Fig. 2. Changes of the bond indices $\left(\mathrm{E}_{\mathrm{AB}}\right)$ and the distribution of the unpaired electron. the $\mathrm{C}-\mathrm{C}$ and $\mathrm{C}-\mathrm{N}$ bonds, while $\longrightarrow$ represent the distributions on the aminomethylene and methyl groups, respectively (CNDO/2). 
ethylamine ion, there are two kinds of cleavage, which one may be able to extend to general cases. If one notices the formal movement of the unpaired electron involved in the reaction, all simple cleavage reactions of doublet ion, which produce a pair of a cation and a radical, can be classified into two characteristic groups; Type I reactions are those reactions in which throughout the reaction the location of the unpaired electron remains in the initial site, while Type II reactions are those reactions in which the unpaired electron changes its location. Such fragmentations may be depicted as follows;

$$
\begin{array}{ll}
{[\mathrm{P}-\dot{\mathrm{Q}}]^{+} \longrightarrow \mathrm{P}^{+}+\cdot \mathrm{Q}} & \text { Type I Reactions } \\
{[\dot{\mathrm{P}}-\mathrm{Q}]^{+} \longrightarrow \mathrm{P}^{+}+\cdot \mathrm{Q}} & \text { Type II Reactions }
\end{array}
$$

where the $\mathrm{P}^{+}+\cdot \mathrm{Q}$ state is assumed to be the ground state, i. e., the lowest electronic state, after complete reaction.

Suppose the excited states of the molecular ion, $[\dot{\mathrm{P}}-\mathrm{Q}]^{+}$and $[\mathrm{P}-\dot{\mathrm{Q}}]^{+}$, of Type I and II reactions, respectively. After the dissociation, the $\mathrm{P} \cdot+\mathrm{Q}^{+}$state is appearently the excited state to the $\mathrm{P}^{+}+\cdot \mathrm{Q}$ state. Since the fragmentation is assumed to be an adiabatic, the correlation diagram of electronic structures for Type I and II reactions can be written as follows;

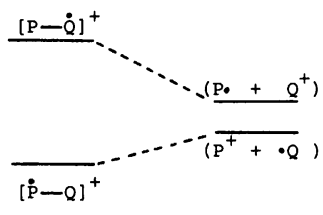

Type I Reactions

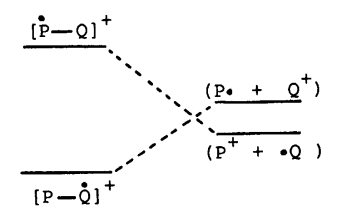

Type II Reactions
Striking difference between Type I and II reactions is that in the latter reaction there appears an interstate crossing, where these two states may interact each other to produce an energically far lower state. According to the perturbation theory, the density of interaction between two states, $S_{0}$ and $S_{1}$, is proportional to, $\mathrm{H}_{01}{ }^{2} / \mathrm{H}_{11}-\mathrm{H}_{00}$ ), where $\mathrm{H}_{00}$ and $\mathrm{H}_{11}$ are the state energies for $\mathrm{S}_{0}$ and $\mathrm{S}_{1}$, respectively ${ }^{30}$. $\mathrm{H}_{01}$ represents matrix element between $S_{0}$ and $S_{1}$ for the total Hamiltonian. Therefore, near the transition states of Type II reactions the high densities of interaction between the ground and the excited states are much expected to cause lower activation energies.

In the cleavage of the $\mathrm{C}-\mathrm{N}$ bond, the unpaired electron remains on the initial fragment. Therefore, this reaction belongs to Type I reactions, in which the stabilization at the transition state by interstate interactions is not much expected to be given, since there do not appear the states which become close in the dissociation.

It may be easy to distinguish Type II reactions from arbitrary fragmentation reactions if we apply the Stevenson's rule ${ }^{311}$ which states that in the fragmentation of a molecular ion such as $[\mathrm{P}-\mathrm{Q}]^{\cdot+} \rightarrow \mathrm{P}^{+}+\cdot \mathrm{Q}$, the positive charge remains predominantly on the fragment with the lower ionization potential (Ip). The Ip's of radicals can be measured or calculated rather accurately by semi-empirical MINDO/ 2 method and some data are available ${ }^{27,32.33)}$. And thus one may understand that most cleavages socalled $\alpha$-cleavage or $\beta$-bond cleavage belong to Type II reactions. It should be pointed out that the high density of the interstate interaction in the $\mathrm{C}-\mathrm{C}$ dissociation of the ethylamine ion is the cause that bring the lower activation energy of the experiment than that of the calculation by the unrestricted MINDO/2 method without CI's.

\section{On the Inadaptability of Bond Index of Intact} Molecular Ion. We may now reach the state that we could interpret the over-all behavior of the molecular ion of ethylamine. First we will give an interpretation to the negative results of the approach by our bond-index method. Being picked up for a specific relative bond-energy 
$\left(E_{\mathrm{xy}}\right)$ in eq. 3, the total energy $(\mathrm{E})$ can be formally expressed in a linear form;

$$
\begin{aligned}
& \mathrm{E}=\mathrm{E}_{\mathrm{XY}}+\mathrm{Z} \\
& \mathrm{Z}=\Sigma \mathrm{E}_{\mathrm{A}}+\sum_{\mathrm{A}>\mathrm{B}} \mathrm{E}_{\mathrm{AB}} \quad(\mathrm{X}, \mathrm{Y} \neq \mathrm{A}, \mathrm{B})
\end{aligned}
$$

Unless the dissociation between atoms $\mathrm{X}$ and $\mathrm{Y}$ changes the electronic structure of the system except for the region around the atoms, $\mathrm{X}$ and $\mathrm{Y}$, the cleavage of the bond with the lower value of $E_{x y}$ produces the higher total energy of system after the dissociation. Therefore, the $E_{x y}$ value may represent the relative depth of the "valley" of the vertical potential energy curve between X and Y (Fig. 3). ·However, actual reaction may undergo electronically and configurationally in an adiabatic manner. If the system has the excited state which follows the potential energy curve as shown $c$ in Fig. 3, new states as shown $a$ whose energies are lower than $b$ and $c$ would be formed by the interstate interactions between $b$ and $c$.

Interstate interactions are symmetry-dependent. Organic molecular ions in general are of very low symmetry because of free rotations around single bonds and most often possessing no element of symmetry. In such ions all MO's are necessarily of the same symmetry species since there is only one. One can not predict this possibility of interstate interaction using the calculated bond energies of the intact molecular ion; this may be the reason of the inadaptability of the bond-indeces.

\section{An Interpretation of the Relationship between} the Net Charge Density of the HO Orbital and the Scission Probability. Hirota et al. have long insisted on the relationship of the scission probability and the net charge density basing on the calculation by means of the LCBO (Linear Combination of Bond Orbital) method ${ }^{10 \sim 12)}$. Recently they have shown that this is also valid in the LCAO (Linear Combination of Atomic Orbital) method ${ }^{(3)}$. Accordingly the relationship is considered not to depend on the type of MO theory but to depend on the net charge density by the HO orbital. Although the exact characteristics of the HOMO of the neutral molecule is not of course the same as that of the ion, we believe that in the first-order approximation, these are regarded as the same. For the reason mentioned in the introduction, the solution of this problem may be necessary even if it is very

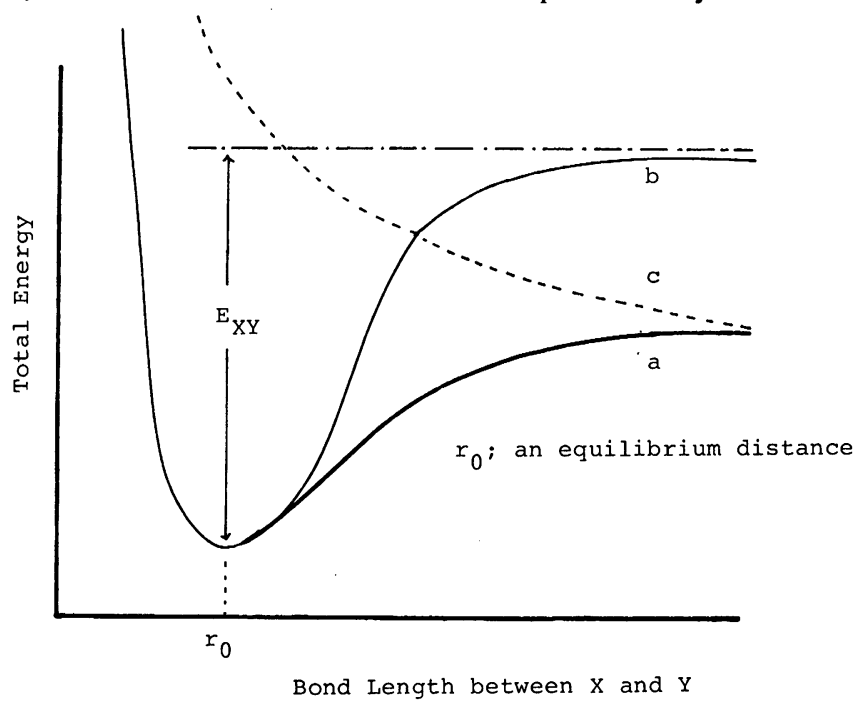

Fig. 3. The relationship between the bond-index and the potential energy curve. 
qualitative.

As have been discussed, calculation of the electronic structure of the ground-state ethylmine ion indicates that the $\alpha$-cleavage reaction of the ethylamine ion belongs to Type II reactions. If the HOMO of the aminomethylene radical and methyl radical are written as $\phi_{\mathrm{CH}_{2} \mathrm{NH}_{2}}$ and $\phi_{\mathrm{CH},}$, respectively, then the HOMO $(\Phi)$ and its antibonding MO $\left(\Phi^{*}\right)$ of the molecular ion may mainly contributed by $\phi_{\mathrm{CH}_{2} \mathrm{NH}_{2}}$ and $\phi_{\mathrm{CH}}$, and expressed as,

$$
\begin{aligned}
& \Phi=a \phi_{\mathrm{CH}_{2} \mathrm{NH}_{2}}+b \phi_{\mathrm{CH}_{3}}+\cdots \\
& \Phi^{*}=b^{\prime} \phi_{\mathrm{CH}_{2} \mathrm{NH}_{2}}+a^{\prime} \phi_{\mathrm{CH}}+\cdots
\end{aligned}
$$

where, $a, b, a^{\prime}$, and $b^{\prime}$ are the coefficients of weight and $a \simeq a^{\prime}$ and $b \simeq b^{\prime}$ may roughly hold.

The unpaired electron is predominantly localized on the aminomethyl site of molecular ion to indicate the relationship, $|a|>|b|$. In the excited configuration, $\left(\dot{\mathrm{C}} \mathrm{H}_{3}-\mathrm{CH}_{2} \mathrm{NH}_{2}\right)^{+}$, which correspends to the $(\dot{\mathrm{P}}-\mathrm{Q})^{+}$state of Type II reactions, the contribution of $\Phi^{*}$ may be the most important. Our previous paper describes the relative bond energies of some excited states of alkylamine ions which are obtained by the extended Hückel method ${ }^{25)}$; the results for the ethylamine ion are quoted in Table 2. In this table one may see that excitation to the lowest vacant $(\mathrm{LV})$ orbital makes the $\mathrm{C}-\mathrm{N}$ bond antibonding, while that to the next higher orbital to the $\mathrm{LV}$ orbital $(\mathrm{LV}+\mathrm{l})$ makes the $\mathrm{C}-\mathrm{C}$ bond antibonding. As discussed in the paper, removal of one electron from the HO orbital weakens the $\mathrm{C}-\mathrm{C}$ bond, indicating that the electron(s) of the HOMO is distributed bondingly on this bond. Therefore, the HOMO should be regarded as $\Phi$ and the $L V+1$ orbital as $\Phi^{*}$.

Using the MO's as expressed in eq. (7), the partial bond-order of the HOMO between the carbon atoms of methyl and aminomethylene groups may be expressed to be $a b$, which may correspond to the partial bond-order of the HOMO of an LCAO MO method and to the charge density of an LCBO MO method. The value of the net charge density by the HOMO may be the same as the partial electron density by the HOMO and therefore, we obtain a qualitative conclusion that the bond where the highest net charge is distributed corresponds to the bond whose dissociation involves the high density interstate interactions giving the most stable state after dissociation; this may be true in general cases. Concerning this conclusion, the following discussions will generally be valid; the LCBO MO's consist of the linear combination of the bond orbitals. The HOMO of them is most contributed by the bond orbital of the bond whose cleavage give the most stable state after the dissociation. Since the total electron densities by an LCBO MO method as well as an LCAO MO method are not largely different: the bond where the electron of the HOMO is much distributed is to have smaller contributions of the electrons from the lower

Table 2. Relationship between Bond Indices and Electronic Excitations (Extended Hückel Method in eV).

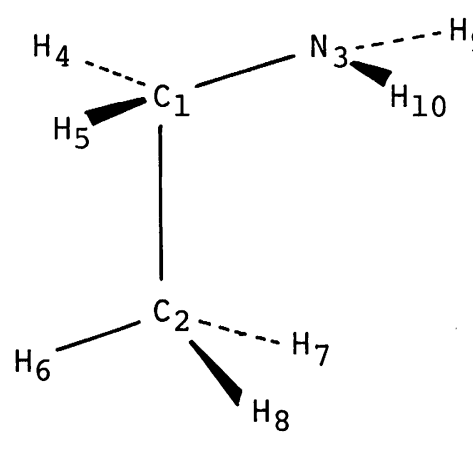

\begin{tabular}{cccccc} 
Bond & Molecule & \multicolumn{1}{c}{ HO } & \multicolumn{1}{c}{ LV } & LV +1 & LV +2 \\
\hline $\mathrm{C}_{1}-\mathrm{C}_{2}$ & -17.23 & 0.75 & 1.88 & 19.55 & 0.35 \\
$\mathrm{C}_{1}-\mathrm{N}_{3}$ & -15.70 & -0.80 & 15.87 & -1.12 & -3.40 \\
$\mathrm{C}_{1}-\mathrm{H}_{4}$ & -21.18 & 0.05 & 0.23 & 1.62 & 1.95 \\
$\mathrm{C}_{2}-\mathrm{H}_{6}$ & -19.82 & 0.02 & 0.33 & 0.29 & 0.03 \\
$\mathrm{C}_{2}-\mathrm{H}_{2}$ & -19.79 & 0.01 & 0.05 & 1.38 & 2.50 \\
$\mathrm{~N}_{3}-\mathrm{H}_{9}$ & -20.79 & -0.01 & 0.71 & 0.34 & 10.30 \\
\hline
\end{tabular}

The bond indices for excited states are recorded as the difference between the molecular and ionic-excited states, i. e., $\mathrm{E}_{\mathrm{AB}}$ (ion) $-\mathrm{E}_{\mathrm{AB}}(\mathrm{mol})$. 
orbitals with the lower energies. Therefore, cleavage of such a bond requires the smaller energy on dissociation. This may be a qualitative reason why the net charge density of the HOMO is proportional to the scission probability.

The Importance of the Stability of the Produced Fragments and the BEP Treatment of Simple Cleavage Reactions. The interstate crossing as appeared in Type II reactions is not always the absolute factor that regulates the rate of reaction; one may see an example in the dissociation of the methyl-t-butylketone ion which undergoes the cleavage between the acetyl and $t$-butyl groups. As Stevenson's rule ${ }^{311}$ shows and as easily verified by experiment, the fragmentation gives a $t$-butyl cation predominantly. The electronic structure of the ground state methyl- $t$-butylketone ion is calculated by the unrestricted INDO method and shown in Fig. 4. In accordance with the organic chemical intui-

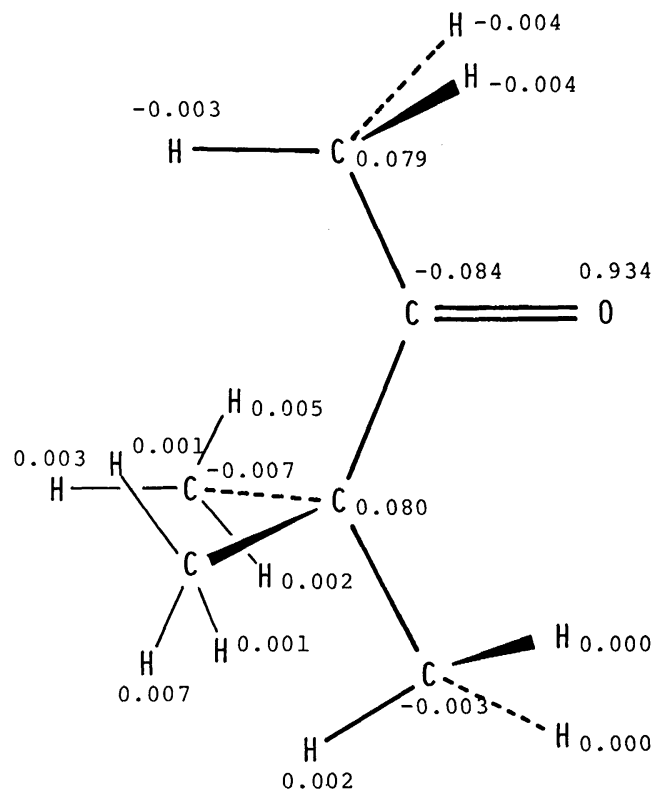

Fig. 4. Spin densities of the methyl- $t$-butylketone ion negative densities represent those with the opposite spin to the considering unpaired electron. tion, the unpaired electron is localized on the oxygen atom, perpendicular to the plane of the $\pi_{\mathrm{co}}$ bond. Therefore, this reaction should be one of Type I reactions.

McLafferty pointed out ${ }^{2)}$ that the most important factor favoring a particular reaction is the stability of the produced system. Stability is important for both ionic and neutral products, although the charge rather than radical is usually found on the fragment which is more favorable for ionization. We recently reported on the relationship between the relative abundance of bond scission and the heat of reaction calculated by the $\mathrm{CNDO} / 2^{34)}$ and MINDO/2 methods ${ }^{32)}$. In spite of uncertainty of the geometries of radicals and ions and of the possible errors due to the adopted MO methods, the agreement was well enough to predict the relative abundance of bond scission. This indicates that most of the reactions of molecular ions are those reactions which take place according to the so-called Bell-Evans-Planyi principle, i. e., the activation energy $(\Delta \mathrm{E})$ is proportional to the heat of reaction $(\Delta \mathrm{H})$ as shown by,

$$
\Delta \mathrm{E}=\mathrm{X} \Delta \mathrm{H}+\mathrm{Y}
$$

where $\mathrm{X}$ and $\mathrm{Y}$ are constant in the same system.

The existence of the chemical reaction with the relationship expressed by eq. (8) was explained by Bell ${ }^{35)}$ and Evans and Polanyi ${ }^{36)}$ and recently reviewed by Dewar ${ }^{37 !}$. Similar discussions may apply to the mass spectral cleavage reaction. Suppose that the molecular ion, P-Q$\mathrm{R}$, is subjected to undergo the competitive fragmentations,

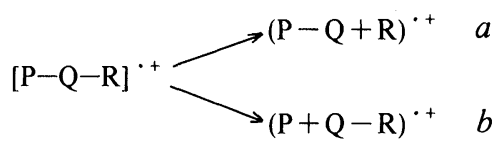

and without loss of generality, we can suppose that the total energy of the $P+Q-R$ state is more stable than that of the $\mathrm{P}-\mathrm{Q}+\mathrm{R}$ state. 


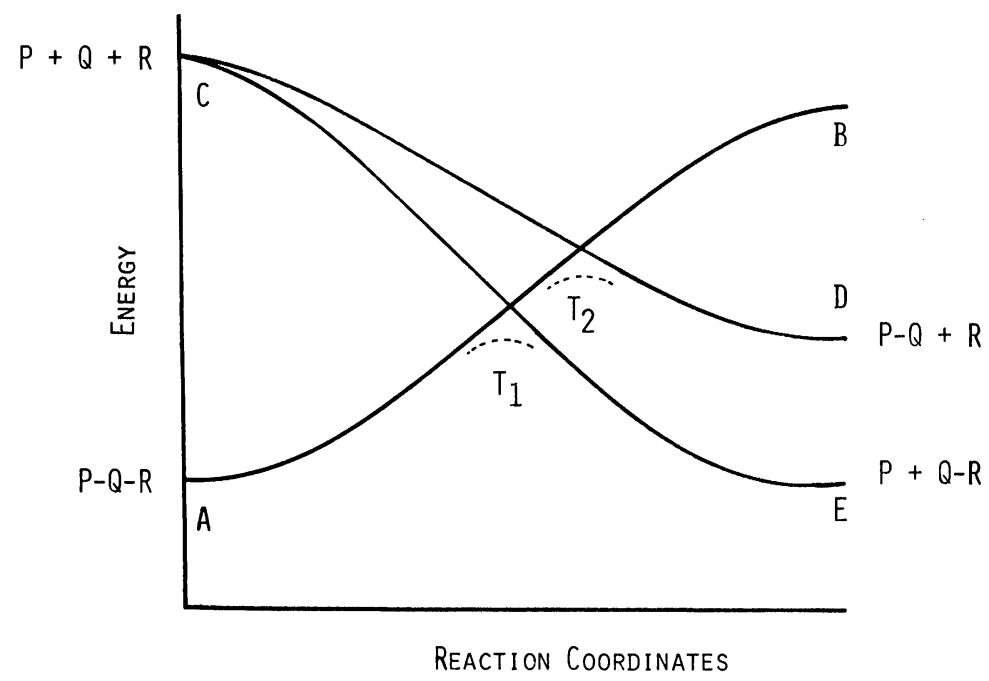

Fig. 5. BEP treatment of simple cleavage reaction.

In this system, the state $C$ in Fig. 5 where the fragments $\mathrm{P}, \mathrm{Q}$, and $\mathrm{R}$ are all separate may have the highest total energy. If the bondlengths between $\mathrm{P}$ and $\mathrm{Q}$, and between $\mathrm{Q}$ and $\mathrm{R}$ of the molecular ion are separated simultaneously to give the state $C$ the potential energy curve of this reaction may be drawn as $\mathrm{AB}$; This is considered to be the highest potential energy curve of this system. If at the state $C$ the fragment $\mathrm{Q}$ is to combine with $\mathrm{P}$ as approaching, the potential energy curve may be drawn as $C D$, while if $Q$ is to combine with $R$, the curve, CE, may be drawn. At the crossing point $\mathrm{Tl}$, the two states become energically equivalent. And therefore, the crossing point $\mathrm{T} 1$ and similarly $\mathrm{T} 2$ are regarded as the highest transition states for the reactions $a$ and $b$, respectively. The reactions of $a$ and $b$ may be switched from the line $A B$ to the lines $C D$ and $C E$, respectively. Of course in the actual reactions, the transition states are lower than $\mathrm{T} 1$ and $\mathrm{T} 2$ since there is little possibility that the bond lengths of the $\mathrm{P}-\mathrm{Q}$ and $\mathrm{Q}-\mathrm{R}$ are stretched simultaneously.

We next consider the possibility that the $\mathrm{CD}$ and $\mathrm{CE}$ cross each other after passing the transition states. There are three cases; 1) the the reactions both $a$ and $b$ do not involve the formations of a $\pi$ bond, 2) the both reactions involve the formations of a $\pi$ bond, and 3 ) only the $a$ reaction is accompanied by the formation of a $\pi$-bond. If the reactions are similar, i. e., cases 1 and 2, we may expect that the shapes of the potential energy curves, $C D$ and $C E$, are similar. Besides, there involve no solvent effects in mass spectral reactions which are important in chemical reactions in solvent. Therefore, in the cases 1 and 2 there would be little possibility that $\mathrm{CD}$ and $\mathrm{CE}$ cross each other. In the case 3 , the formation of bond is considered not to occur abruptly near the final state but to occur gradually as the reaction proceeds. This causes the energy of the transition state of the reaction which forms the $\pi$-bond lower than that of the reaction which does not. Thus it may be concluded that almost all of simple cleavage reaction of the doublet molecular ions are those reactions which occur according to the BEP principle.

\section{Acknowledgment}

The computations were carried out on 


\section{An Interpretation of Behavior of Ethylamine Ion Produced by Electron-impact}

HITAC $8800 / 8700$ computers at the computation center of the University of Tokyo.

\section{References}

1) An MO approach to the interpretation of organic mass spectra. VIII. Part VII. H. Ichikawa and M. Ogata, Bull. Chem. Soc. Japan, 47, 2591 (1974).

2) F. W. McLafferty, Ed., "Mass Spectrometry of Organic Ions," Academic Press, New York, N. Y. 1963.

3) F. W. McLafferty, "Interpretation of Mass Spectra," W. A. Benjamin, New York, N. Y., 1966.

4) H. Budzikiewicz, C. Djerassi, and D. H. Williams, "Mass Spectrometry of Organic Compounds," Holden-Day, San Francisco, Calif., 1967.

5) R. I. Reed, Ed., "Modern Aspect of Mass Spectrometry," Plenum Press, New York, N. Y., 1968, pp. 143-168.

6) H. M. Rosenstock, M. B. Wallenstein, A. L. Wahrhaftig, and H. Eyring, Proc. Nat. Acad. Sci. U. S., 38, 667 (1952).

7) H. M. Rosenstock and M. Krauss, Adv. Mass Spectrom., 2, 251 (1963).

8) H. M. Rosenstock, ibid., 4, 523 (1968).

9) R. Thompson, "Applied Mass Spectrometry," Inst. of Petroleum, London, 1954, p. 154.

10) K. Hirota M. Itoh, Bull. Chem. Soc. Japan, 38, 599 (1965).

11) K. Hirota and J. Takezaki, Bull. Chem. Soc. Japan, 41, 76 (1968).

12) K. Hirota and Y. Niwa, J. Phys. Chem., 72, 5 (1968).

13) K. Hirota, I. Fujita, M. Yamamoto, and Y. Niwa, J. Phys. Chem., 74, 410 (1970).

14) J. C. Lorquet, Mol. Phys., 9, 101 (1965).

15) C. Krier, J. C. Lorquet, and A. Berlingin, Org. Mass. Spectrom., 8, 387 (1974).

16) H. Ichikawa and M. Ogata, Mass Spectrosc. (Japan), 21, 91 (1973).

17) M. Tsuchiya and Y. Hori, Mass Spectrosc. (Japan), 22, 79 (1974)

18) J. C. Leclerc and J. C. Lorquet, J. Phys. Chem., 71, 787
(1967).

19) J. A. Pople, D. P. Santry, and G. A. Segal, J. Chem. Phys., 43, S129 (1965); J. A. Pople and G. A. Segal, ibid., 43, S136 (1965)

20) J. A. Pople and G. A. Segal, J. Chem. Phys., 44, 3289 (1965).

21) J. A. Pople, D. L. Beveridge, and P. A. Dobosh, J. Chem. Phys., 47, 2026 (1967).

22) N. C. Baird and M. J. S. Dewar, J. Chem. Phys., 50, 1262 (1969).

23) M. J. S. Dewar and E. Haselbach, J. Amer. Chem. Soc., 92, 590 (1970); ibid., 92, 3854 (1970).

24) H. Fischer and H. Kollmar, Theoret. Chim. Acta (Berl.), 16, 163 (1970).

25) M. Ogata and H. Ichikawa, Bull. Chem. Soc. Japan, 45, 3231 (1972).

26) O. Kajimoto, Ph. D. Thesis, Osaka University, Osaka, Japan, 1971; see also refs. 22 and 23.

27) H. Ichikawa and M. Ogata, Bull. Chem. Soc. Japan, 47, 2591 (1974)

28) J. Collin, M. J. Franskin, and D. Hyatt, Bull. Soc. Roy. Sci. Liege, 36, 318 (1967).

29) W. A. Chupka, J. Chem. Phys., 30, 191 (1959).

30) e. g., R. McWeeny and B. T. Sutclifle, "Methods of Molecular Quantum Mechanics," Academic Press, London, England, 1969, Chapter 2.

31) D. P. Stevenson, Discuss. Faraday Soc., 10, 35 (1951).

32) H. Ichikawa and Ogata, Chem. Pharm. Bull. (Tokyo), 22, 1560 (1974).

33) A. G. Harrison, ref. 1. Chapter 5.

34) H. Ichikawa and M. Ogata, Bull. Chem. Soc. Japan, 46, 1873 (1973).

35) R. P. Bell, Proc. Roy. Soc., Ser. A, 154, 414 (1936).

36) M. G. Evans and M. Polanyi, Trans. Faraday Soc., 32, 1340 (1936).

37) M. J. S. Dewar, "The Molecular Orbital Theory of Organic Chemistry," McGraw-Hill, Inc., New York, N. Y. 1969, Chapter 8. 